\title{
Time variations of the ON2 water maser spectrum during 1981-1995
}

\author{
E.E. Lekht ${ }^{1,2}$, A. Marquez ${ }^{1}$ and J.E. Mendoza-Torres ${ }^{1}$ \\ 1 Instituto Nacional de Astrofisica, Optica y Electronica, Apartado Postal 51 y 216, Puebla, Pue., Z.P. 72000, Mexico \\ 2 Sternberg Astronomical Institute, Universitetsky prospect 13, 19899, Moscow, Russia
}

Received January 3; accepted May 6, 1996

\begin{abstract}
Results of observations at $22.2 \mathrm{GHz}$ of the water maser source ON2 carried out at the Pushchino RT-22 radio telescope during 1981-1995 are presented. The long-time regular observations of ON2 show that the time interval between the two high levels of the integrated $\mathrm{H}_{2} \mathrm{O}$ flux density is more than 20 years. The flare component of the variability of total flux as well as strong flares of some features were not detected. The emission is concentrated, basically, in two spectrally separated groups of features. The observed anticorrelation of the fluxes of these groups was manifested only in the period of minimum activity of the $\mathrm{H}_{2} \mathrm{O}$ maser. It seems that the transition of the maser takes place from the unsaturated state to a partly saturated one and vice versa. The variability of the maser emission has a global character for ON2 and may be caused by non-stationary physical processes at the early stages of stellar evolution.
\end{abstract}

Key words: ON2 - masers - radio lines: ISM

\section{Introduction}

The water maser source $\mathrm{ON} 2$ is located in a region of star formation in the direction of Cygnus $\mathrm{X}$, a known complex of molecular clouds. The $\mathrm{H}_{2} \mathrm{O}$ maser was first detected by Johnston et al. (1973). Later observations (Cato et al. 1976; Genzel \& Downes 1977) have shown that the emission comes from two spatially distinct sources.

The source ON2 is elongated north-south including three regions: northern, central and southern parts, named $\mathrm{ON} 2(\mathrm{~N}), \mathrm{ON} 2(\mathrm{C})$ and $\mathrm{ON} 2(\mathrm{~S}) \cdot \mathrm{H}_{2} \mathrm{O}$ maser emission is associated with the central and southern parts (Fig. 1).

This region has been extensively mapped in the ${ }^{12} \mathrm{CO}$ and ${ }^{13} \mathrm{CO}$ molecular lines (Matthews et al. 1986) and in $\mathrm{NH}_{3}$ and $\mathrm{HCO}^{+}$molecular lines (Dent et al. 1988). In the central part of $\mathrm{ON} 2$ the positions of the maxima of the ${ }^{13} \mathrm{CO}$ and $\mathrm{NH}_{3}$ emission coincide with each other. In the southern part there is another molecular cloud observed in ${ }^{12} \mathrm{CO}, \mathrm{NH}_{3}$ and $\mathrm{HCO}^{+}$lines. The observations of the molecular clouds have shown that the small scale structures in ON2 are associated with star formation and have masses $\sim(0.3-1) 10^{4} M_{\odot}$ (Matthews et al. 1986). According to Dent et al. (1988) $\mathrm{NH}_{3}$ and $\mathrm{HCO}^{+}$observations have revealed that the region, including $\mathrm{ON2}(\mathrm{C})$ and $\mathrm{ON} 2(\mathrm{~S})$, contains two massive rotating cloud cores with masses of $\approx 300 M_{\odot}$.

The distribution of ionized hydrogen in ON2 region was observed at $\lambda=6 \mathrm{~cm}$ (Matthews et al. 1973;

Send offprint requests to: J.E. Mendoza-Torres
Harris 1976) and $11 \mathrm{~cm}$ (Matthews \& and Spoelstra 1983). The maximum of continuum emission (H II C) is located between two molecular cloud cores. At the north of $\mathrm{H}$ II $\mathrm{S}$ there is the compact region $\mathrm{H}$ II $\mathrm{C}$ which is within the molecular cloud core at $\mathrm{ON} 2(\mathrm{C})$. There is strong $\mathrm{OH}$ maser emission near them (Elldér et al. 1969; Norris et al. 1980).

Thus, the main $\mathrm{H}_{2} \mathrm{O}$ maser (named $\mathrm{N}$ component), the $\mathrm{OH}$ maser as well as the compact $\mathrm{H}$ II region are located near the ${ }^{13} \mathrm{CO}$ maximum. The other $\mathrm{H}_{2} \mathrm{O}$ maser ( $\mathrm{S}$ component) is at a distance of 80 arcsec from the main component and it is associated with a maximum in the continuum emission and with the minimum of the CO emission. The observations carried out by White \& Macdonald (1979) have shown that the $\mathrm{H}_{2} \mathrm{O}$ maser emission has strong time variations.

VLBI aperture synthesis observations of $\mathrm{H}_{2} \mathrm{O}$ maser emission has been carried out by Walker et al. (1978). The synthesis map of $\mathrm{ON} 2$ shows two regions of $\mathrm{H}_{2} \mathrm{O}$ emission. For an assumed distance of $5.5 \mathrm{kpc}$ the regions are each of about $10^{15} \mathrm{~cm}$ in size and are separated by about 2.7 $10^{15} \mathrm{~cm}$. The separation of the features is suggestive of a shell (Walker et al. 1978).

The distance to ON2, determined by different methods, varies between 900 pc (Campbell et al. 1982; Turner \& Forbes, 1982; Lozinskaya et al. 1994) and $5.5 \mathrm{kpc}$ (Riefenstein et al. 1970; Palagi et al. 1993). The distance of $D=5.5 \mathrm{kpc}$ is adopted here, although a distance of 900 $\mathrm{pc}$ is still possible. The $900 \mathrm{pc}$ distance may be assumed if 


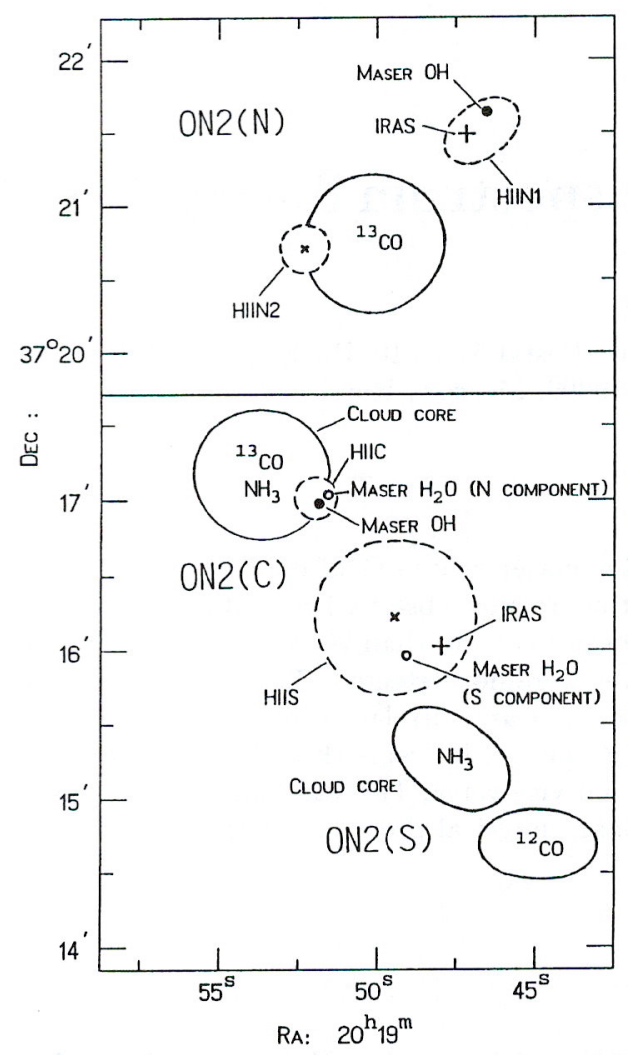

Fig. 1. Map showing the positions of molecular clouds (solid line), the HII regions (the dashed line), the $\mathrm{H}_{2} \mathrm{O}$ and $\mathrm{OH}$ maser sources and IK sources in the ON2 region

ON2 is associated with the local molecular cloud (Campbell et al. 1982; Pitault 1981). If one assumes $D=6 \mathrm{kpc}$, then the compact region $\mathrm{H}$ II C must be excited by a B0.8 type young star (Dent et al. 1988).

The aim of our long-term monitoring of the $\mathrm{H}_{2} \mathrm{O}$ maser sources in the ON2 region is the study of long-term variability of the flux as well as the drift of the velocity centroid of $\mathrm{H}_{2} \mathrm{O}$ spectra.

\section{Observations}

The observations of $\mathrm{H}_{2} \mathrm{O}$ maser emission in $\mathrm{ON} 2$ were carried out during 1981-1995, using the RT-22 radio telescope of the Radio Astronomical Station of the Lebedev Physical Institute in Pushchino, Russia. The time interval between two consecutive observational runs was about 2 months.

At the frequency of $22 \mathrm{GHz}$ the beam width of the $22-\mathrm{m}$ antenna is $2.6 \mathrm{arcmin}$. The noise temperature of the receiving system was about $200 \mathrm{~K}$. An antenna temperature of $1 \mathrm{~K}$ from a point source with unpolarized emission corresponds to a flux density of $25 \mathrm{Jy}$.

A radiometer with a maser amplifier was used. From the end of 1993 a transistor amplifier with low noise was used. The radiometer works on the principle of symmet- rical beam modulation, by switching alternately between two identical feedhorns directed to two adjacent regions on the sky.

The signal was analyzed with a 96-channel filter-bank spectrometer with a resolution of $7.5 \mathrm{kHz}(0.1 \mathrm{~km} / \mathrm{s}$ in radial velocity at $22 \mathrm{GHz}$ ). Sufficient bandwidth for the analysis was obtained by three changes of the local oscillator frequency.

Observations of the source ON2 were made using the "On-On" method. In this method the antenna is directed to the source first by one feedhorn and then by the other one. If the emission of the source has some linear polarization, then variation between the polarization planes of the emission from the source and that of the antenna feedhorn may lead to change in the observed flux. To avoid this effect, ON2 was observed always at approximately the same position angles. The integration time of each record was about $20 \mathrm{~min}$. A detailed description of the apparatus and the observation technique is given in Sorochenko et al. (1985) and Lekht et al. (1995).

The data were reduced at the National Institute of Astrophysics, Optics and Electronics, Tonantzintla, Puebla, Mexico.

\section{Presentation of the results}

ON2 is a double source, with the principal emission originating from the $\mathrm{N}$ maser component and the $\mathrm{S}$ component having only weak $\mathrm{H}_{2} \mathrm{O}$ emission at velocities $<-8 \mathrm{~km} \mathrm{~s}^{-1}$. For this reason, we carried out observations in the direction of the maximum $\mathrm{H}_{2} \mathrm{O}$ emission, where the principal maser source (R.A. $=20^{\mathrm{h}} 19^{\mathrm{m}} 51.8^{\mathrm{s}}$, Dec. $\left.=37^{\circ} 17^{\prime}\right)$ is situated. Due to the beam response the flux density of the $\mathrm{S}$ component was further attenuated.

The catalog of $86 \mathrm{H}_{2} \mathrm{O}$ spectra of $\mathrm{ON} 2$ is presented in Figs. 2a-j. These spectra were obtained during 19811995 with a spectral resolution of $0.1 \mathrm{~km} \mathrm{~s}^{-1}$. In Fig. $2 \mathrm{k}$ is shown the ON2 emission in the velocity range, $-10<$ $V_{\mathrm{LSR}}<-5 \mathrm{~km} \mathrm{~s}^{-1}$, which was detected during 1981-1983. In the period of minimum maser activity (1984-1992) we did not observe emission in this part of the spectrum.

The variations of total flux from 1972 (the discovery of $\mathrm{H}_{2} \mathrm{O}$ maser emission in the ON2 region) up to 1995 are given in Fig. 3. We have used data obtained by many authors from 1972 up to 1977 (Johnston et al. 1973; Cato et al. 1976; White \& Macdonald 1979; Genzel \& Downes 1979). For computations of total flux for this interval, we did not include the contribution of the southern component. The dashed line represents the smoothed curve. The strong variations of the total flux as compared with this smoothed curve reflect the real changes of $\mathrm{H}_{2} \mathrm{O}$ spectra. In spite of the lack of observations between 1977 and 1981, the flux is clearly decreasing (down to a minimum level of $\approx 200 \mathrm{Jy} \mathrm{km} \mathrm{s}^{-1}$ ). This value, at an average, was kept during 1985-1991. Beginning from 1992, a fast increase of the emission takes place and at the end of 1995 the total 

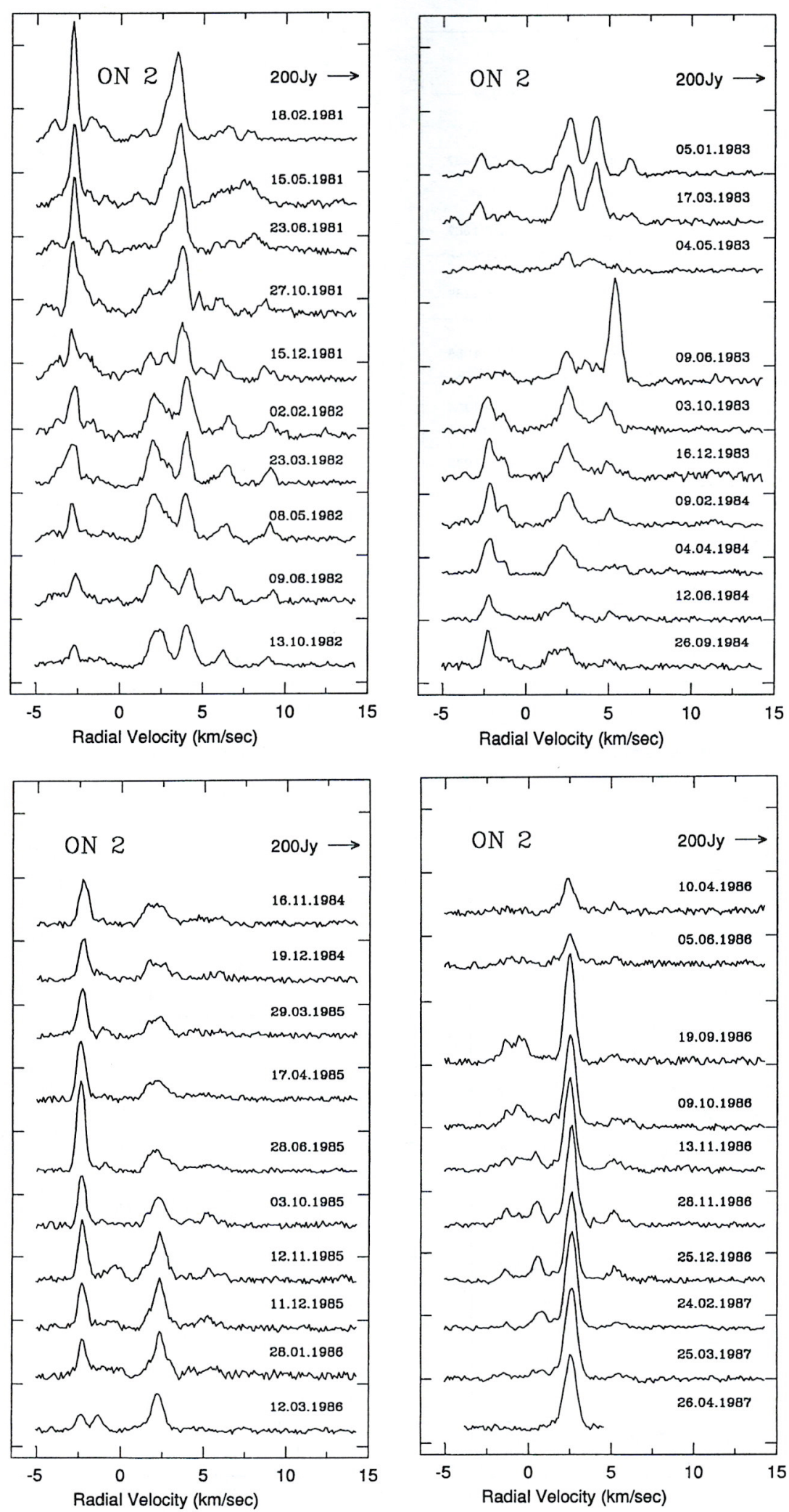

Fig. 2. a-k) Spectra of $\mathrm{H}_{2} \mathrm{O}$ maser emission of $\mathrm{ON} 2$ from 1981 to 1995 . Fluxe interval shown on the vertical axis is $200 \mathrm{Jy}$, except in $(\mathrm{k})$ where it is $50 \mathrm{Jy}$ 

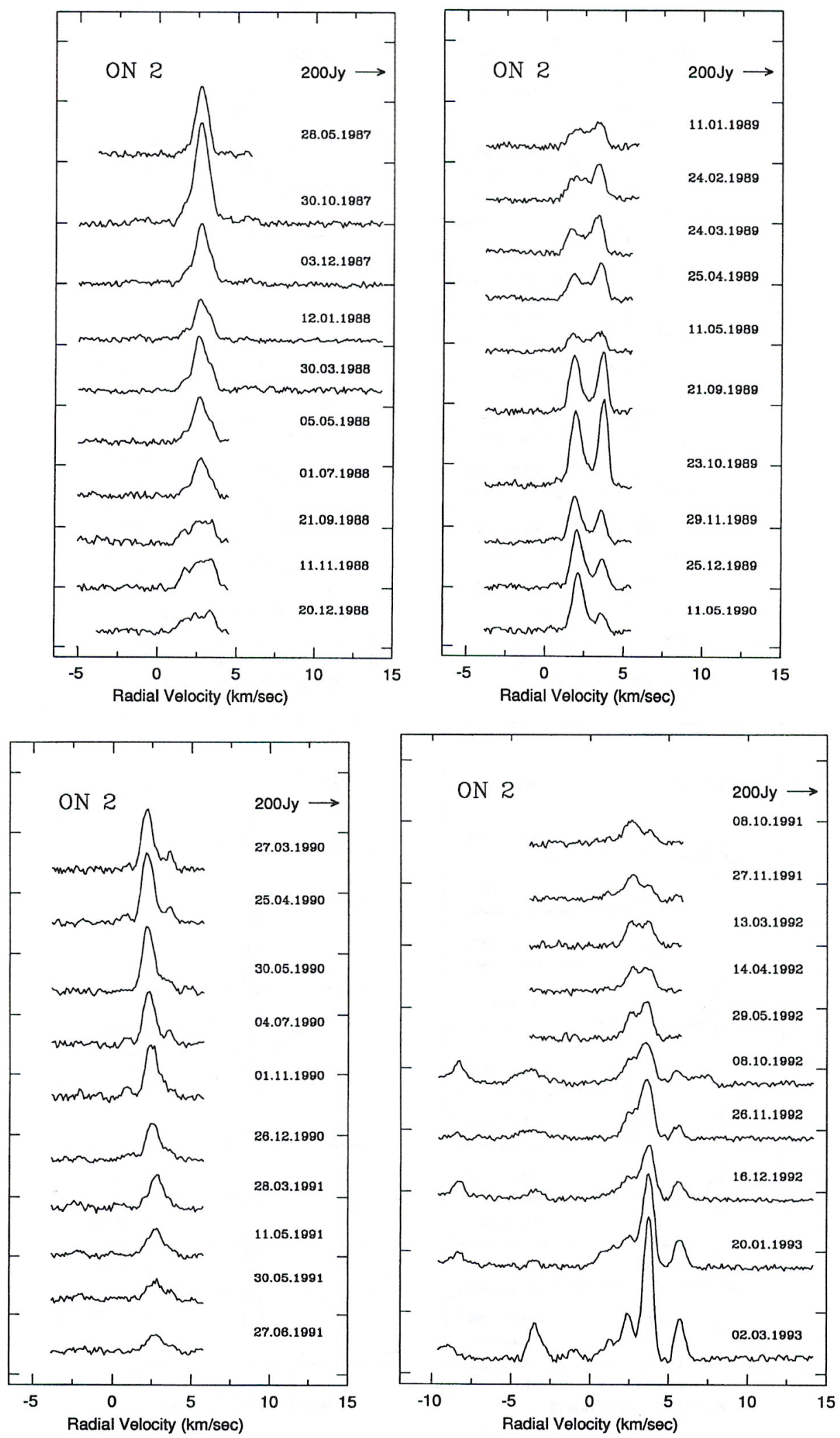

Fig. 2. continued 

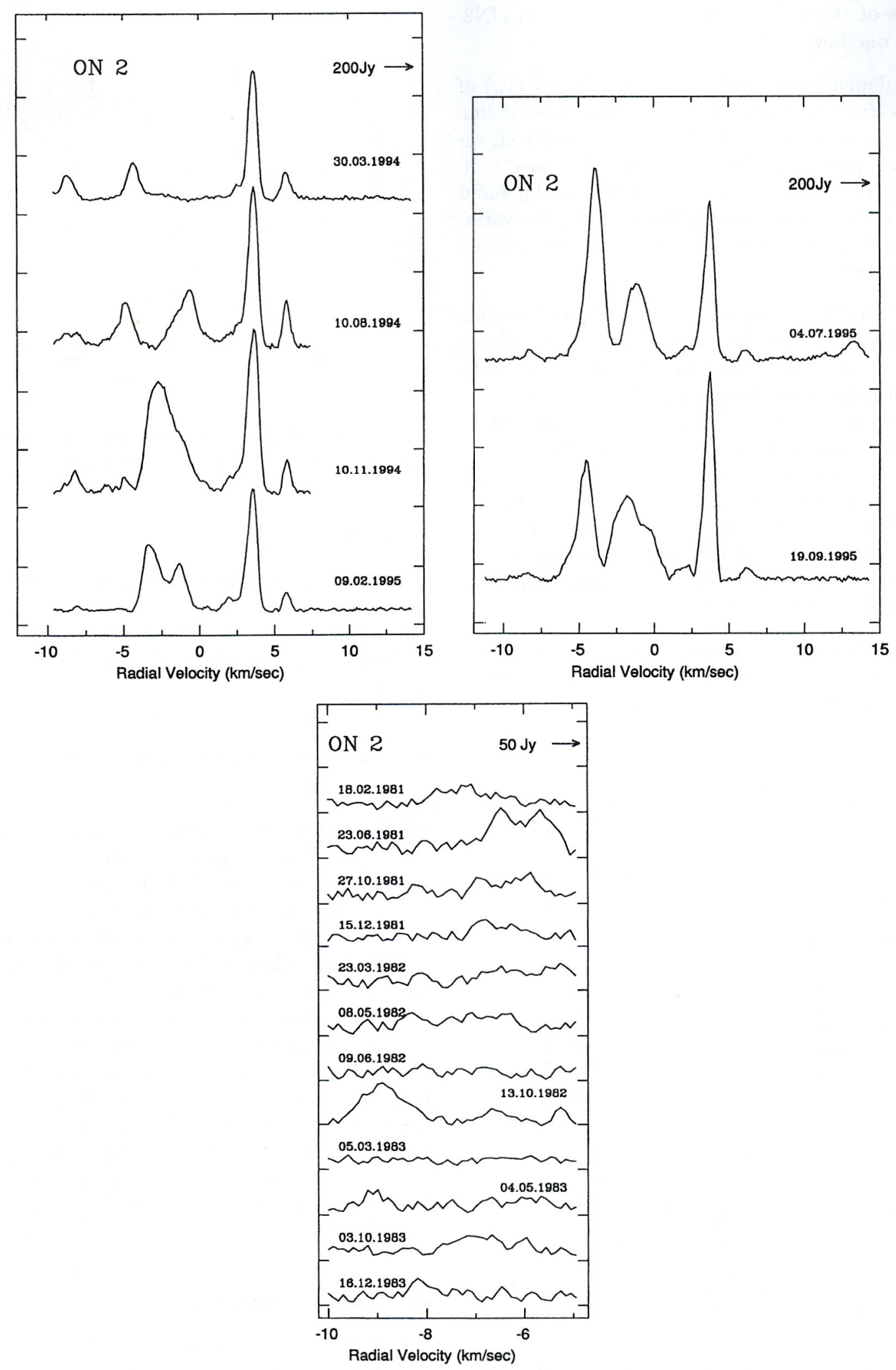

Fig. 2. continued 
flux attains $1500 \mathrm{Jy} \mathrm{km} \mathrm{s}^{-1}$. Thus, there were three different periods of the activity of the $\mathrm{H}_{2} \mathrm{O}$ maser in $\mathrm{ON2}$ : two high and one low.

The time dependence of the centroid velocity $\left(V_{0}\right)$ of the $\mathrm{H}_{2} \mathrm{O}$ spectrum is shown in Fig. 4. The dashed line represents the smoothed time variation of the centroid velocity. The most probable value of $V_{0}$ is 2 to $2.5 \mathrm{~km} \mathrm{~s}^{-1}$. In 1986 and 1995, two minima of $V_{0}$ were observed of 0.4 and $-0.6 \mathrm{~km} \mathrm{~s}^{-1}$ respectively. Correlation between the variations of the total flux of all the spectrum and the centroid velocity is not seen.

Mean spectra (Fig. 5) for these three different periods of maser activity and for the total period of our observations were obtained. It is seen that the emission peaks are situated principally in two parts of the spectrum: from -5 up to 0 and from 0 up to $7 \mathrm{~km} \mathrm{~s}^{-1}$. The total fluxes were calculated separately for these parts of the spectra; their time variations are shown in Fig. 6. In each group the emission was at some fixed radial velocities during any given period. Such velocities are -2.3 and $-0.9 \mathrm{~km} \mathrm{~s}^{-1}$ for the first group and $2.5,3.8$ and $5.4 \mathrm{~km} \mathrm{~s}^{-1}$ for the second one. This may also be seen in the averaged spectrum for the whole period of our observations (Fig. 5c).

During the high activity weak emission was observed $V_{\mathrm{LSR}}<-5 \mathrm{~km} \mathrm{~s}^{-1}$ and $V_{\mathrm{LSR}}>7 \mathrm{~km} \mathrm{~s}^{-1}$. The lifetime of such features in the active stage at $V_{\mathrm{LSR}}<-5 \mathrm{~km} \mathrm{~s}^{-1}$ was a few months.

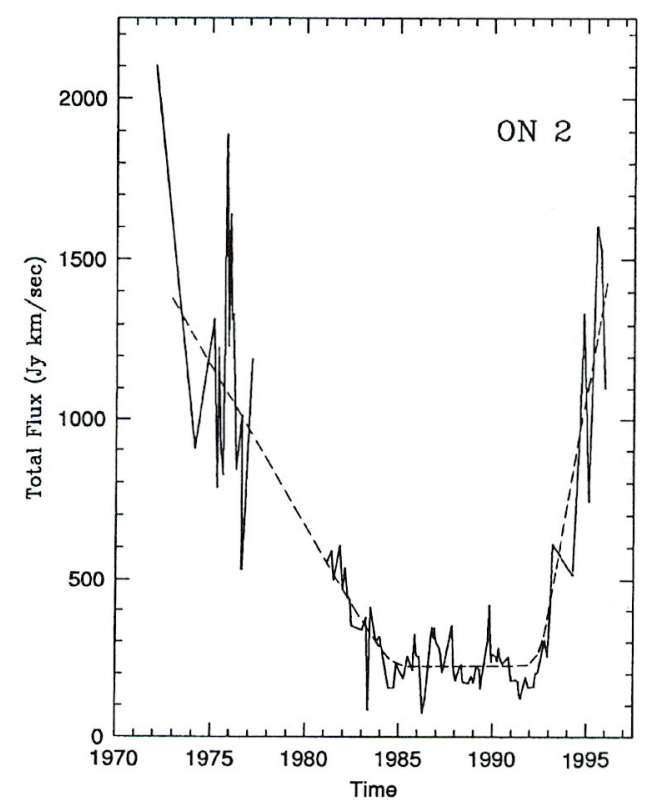

Fig. 3. Total flux evolution of the maser emission of ON2. The dashed line denotes the smoothed curve

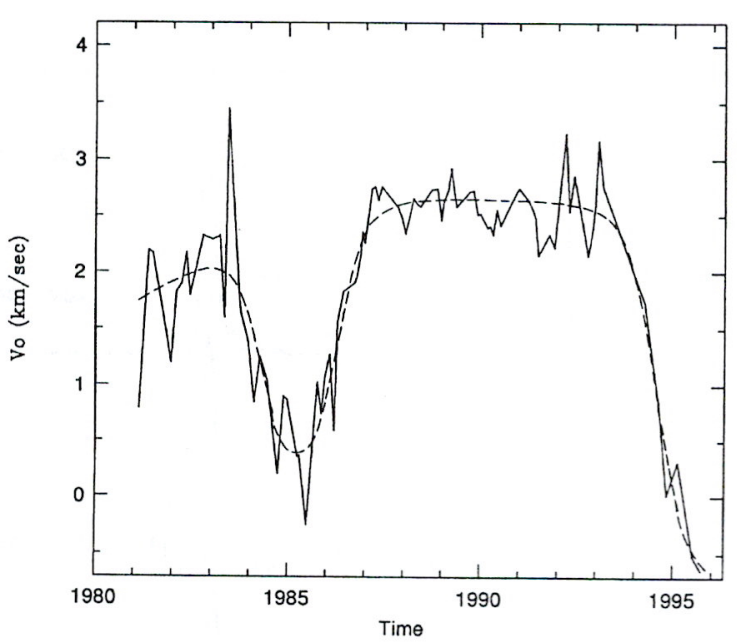

Fig. 4. Time dependence of the centroid velocity $\left(V_{0}\right)$ of the $\mathrm{H}_{2} \mathrm{O}$ spectrum. The dashed line denotes the smoothed curve

\section{Discussion}

Our observations reveal two types of variations of the integrated $\mathrm{H}_{2} \mathrm{O}$ flux: long-term for all $\mathrm{H}_{2} \mathrm{O}$ spectra (dashed line in Fig. 3) and oscillatory for the group of features in the velocity range from 0 to $7 \mathrm{~km} \mathrm{~s}^{-1}$ (dashed line in Fig. 6).

\subsection{Long-time variations of the total flux}

For a number of $\mathrm{H}_{2} \mathrm{O}$ masers associated with regions of star formation the variability of total flux always may be represented as a superposition of long-term and flare components (see Lekht et al. 1995; Berulis et al. 1995). The ON2 source strongly differs from them. The form of the curve in Fig. 3 shows no flare components in ON2 (except in 1989 June when a flare was detected at a velocity of $5.3 \mathrm{~km} \mathrm{~s}^{-1}$ having a flux of $320 \mathrm{Jy}$ ). There are periods of active and relatively "quiet" states of the maser.

Considering that 1972 (discovery of the ON2 maser) and 1995 were the years of its maximum activity then the period of long-term variability of the maser is more than 20 years. In the active period, the increasing of emission takes place in all the velocity ranges. Such variability may be related with non-stationary accretion of a gas onto a star during a stage in its formation (Yorke \& Krügel 1977; Garlick 1978).

\subsection{Anticorrelation of the fluxes}

During 1983-1992, oscillations occured in the integrated flux of the second group $\left(0<V_{\mathrm{LSR}}<7 \mathrm{~km} \mathrm{~s}^{-1}\right)$, with a period of 3-4 years and amplitude around $160 \mathrm{Jy} \mathrm{km}$ $\mathrm{s}^{-1}$ (see Fig. 6). To find the existence of the oscillations beyond this period is very difficult because of the sharp 

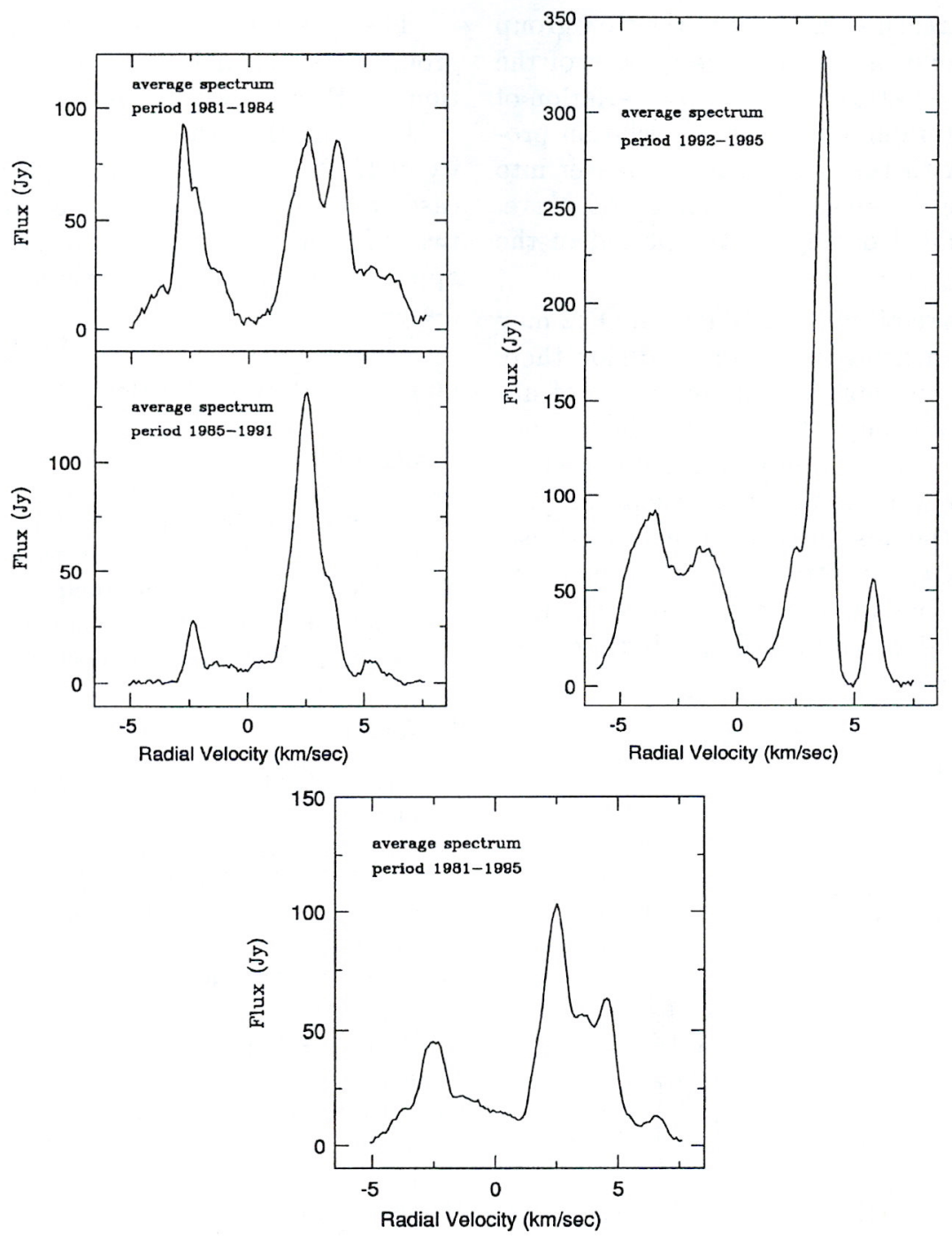

Fig. 5. a-c) The averaged spectra for tree different periods of the maser activity of ON2: a) 1981-1984 and 1985-1991, b) 1992-1995, c) all the period

variations of the flux. Nevertheless, it is quite probable that the time oscillations extend beyond 1983-1993.

In 1984-1986, anticorrelation of the fluxes of the two groups $\left(-5<V_{\mathrm{LSR}}<0\right.$ and $\left.0<V_{\mathrm{LSR}}<7 \mathrm{~km} \mathrm{~s}^{-1}\right)$ was observed. After that, the emission of the first group had strongly decreased. In spite of this circumstance, further flux oscillations of the first group occurred and they were in antiphase relative to the second group. Nevertheless, we do not give any large significance to the fact of the anticorrelation of the fluxes in 1987-1991, because the value of the signal over velocity ranges of -5 to $0 \mathrm{~km} \mathrm{~s}^{-1}$ was below the noise level. In the active phases of the maser (before 1982 and after 1994) the emission of both groups was approximately equal. This is seen also in the general averaged spectrum (Fig. 5c).

\subsection{Interpretation}

The division of the features of each $\mathrm{H}_{2} \mathrm{O}$ spectrum into two basic groups and the existence of the anticorrelation of the fluxes are possible only if these groups are spacially separated and are located in different parts of an envelope. This statement is in a good agreement with the results of VLBI observations (Walker et al. 1978). If the envelope rotates and/or expands the spectral groups will be far one from another as far as the radial velocities. A similar maser structure was observed in W75S(2) (Genzel \& Downes 1977) and possibly in S128 (Berulis et al. 1995), but in $\mathrm{W} 75 \mathrm{~S}(2)$ the total dimension of the envelope is far greater than in ON2.

According to Fig. 4 the curve $V_{0}$ has two minima. The first of them is due to the existence of the flux anticorrelation between the two groups. The second minimum is 
caused by the faster increasing emission of the first group compared with the second one during the growth of the maser activity. Thus, the nature of the strong variation of $V_{0}$ is different. The beginning of the anticorrelation process coincides in time with the transition of a maser into a quiet state, i.e. in the flat part of the smoothed curve. This process has continued during all the period of the minimum maser activity.

The observed anticorrelation of the fluxes in ON2 may be explained as follows. During maximum emission there was sufficient radiative pumping for the existence of unsaturated masers in both groups. In this case there is not competitive radiative modes for pumping. During the long minimum of maser activity, there was insufficient pumping to support the maser in the unsaturated state. In this case, the maser exists in a partly saturated state when there are radiative modes competing for the pumping. Such processes may cause the anticorrelation of the fluxes of two groups of spectral features.

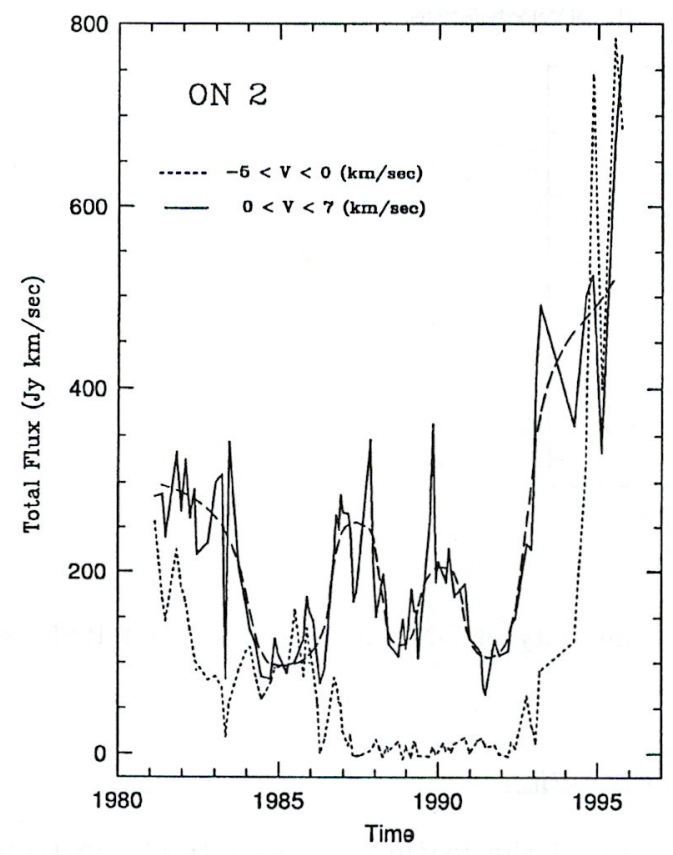

Fig. 6. Time variations of two groups of spectral features. The dashed line denotes the smoothed curve which reflect the flux oscillations of the second group

\section{Conclusions}

The results of the long-term monitoring of $\mathrm{H}_{2} \mathrm{O}$ maser emission in $\mathrm{ON} 2$ are presented. There are two types of variability of the integrated flux: long-term variability, having a time interval between two maxima of more than 20 years, and oscillations with a period of 3-4 years.
The observed anticorrelation of the fluxes of two groups of spectral features requires their spatial separation. VLBI observations suggest such a separation.

It seems that the character of the observed variability of $\mathrm{H}_{2} \mathrm{O}$ emission is related to the central star. In this case, we may say that in the process of star formation two different periods of stellar luminosity variations are apparent, a period of more than 20 years, and a period of 3-4 years.

The results of the study of individual components will be published at a later date.

Acknowledgements. We should like to thank Prof. R.L. Sorochenko, Dr. I.I. Berulis and their associates at the Radio Astronomical Station of the Lebedev Physical Institute for generously providing observing time at the Pushchino RT-22 radio telescope and for their help during the observations. We also thank Dr. G.H. Macdonald for a number of comments which led to the improvement of the paper.

\section{References}

Berulis I.I., Lekht E.E., Mendoza-Torres E., 1995, AZh 72, 468 Campbell M.F., Hoffmann W.F., Thronson Jr.H.A., Niles D., Nawtel R., 1982, ApJ 261, 550

Cato B.T., Rönnäng O.B., Rydbeck O.E.H., Lewin P.T., Yngvesson K.S., Cardiasmenos A.G., Shanley J.F., 1976, ApJ 208, 87

Dent W.R.F., Macdonald G.H., Andersson M., 1988, MNRAS 235,1398

Elldér J.R., Rönnäng B., Winnberg A., 1969, Nat 222, 67

Garlick A.R., 1978, A\&A 68, 113

Genzel R., Downes D., 1977, A\&AS 30, 145

Johnston K.J., Sloanaker R.M., Bologna J.M., 1973, ApJ 182, 67

Harris S., 1976, MNRAS 174, 1

Lekht E.E., Mendoza-Torres E., Sorochenko R.L., 1995, ApJ 443,222

Lozinskaya T.A., Pravdikova V.V. Sitnik T.G., 1994, AZh 71, 515

Matthews H.E., Goss W.M., Winnberg A., Habing H.J., 1973, A\&A 29, 309

Matthews H.E., Spoelstra T.A.T., 1983, A\&A 126, 433

Matthews N., Andersson M., Macdonald G.H., 1986, A\&A 155, 99

Norris R.P., Booth R.S., Davis R.J., 1980, MNRAS 190, 163

Palagi F., Cesaroni R., Comeretto G., Felli M., Natale V., 1993, A\&AS 101, 153

Pitault, 1981, A\&A 97, L5

Reifenstein E.C., Wilson T.L., Burke B.F., Mezger P.G., Altenhoff W.J., 1970, A\&A 4, 357

Sorochenko R.L., Berulis I.I., Gusev V.A., Lekht E.E., Nagornykh L.M., Smirnov G.T., 1985, Trudy FIAN 159, 50

Turner D.G., Forbes D., 1982, PASP 94, 789

Walker R.C., Burke B.F., Haschick A.D., et al., 1978, ApJ 226, 95

White G.J., Macdonald G.H., 1979, MNRAS 188, 745

Yorke H.W., Krügel E., 1977, A\&A 54, 183 